\title{
Origen e historia natural del asma
}

\section{Origin and natural history of asthma}

La Jornada de Primavera de la Sociedad Chilena de Enfermedades Respiratorias realizada el 30 de agosto de 2019, fue dedicada al Asma Bronquial. Esta enfermedad es prevalente en Chile y en el mundo y aunque fue descrita en la antigüedad aún enfrenta problemas en el diagnóstico, manejo y tratamiento debido a su heterogeneidad.

Desde hace más de 30 años la definición de asma bronquial ha sido clínico-fisiopatológica: "historia de síntomas respiratorios como tos, sibilancias, falta de aire, opresión en el pecho que varían con el tiempo y en intensidad junto con limitación variable al flujo aéreo espiratorio"'. Esta definición ha llevado a asumir que el asma bronquial es una sola a pesar del aforismo que "no todo lo que silba en el tórax es asma, pero la mayoría de las veces si lo es", sin identificar las diferentes características de cada uno de los pacientes que la presentan, produciendo en muchas ocasiones un mal tratamiento por un mal diagnóstico.

Cuando se implementó la técnica del lavado bronco-alveolar y de la biopsia de mucosa bronquial mediante la utilización del broncoscopio flexible, fue posible caracterizar el proceso inflamatorio de la vía aérea. En un comienzo se determinó que en la reacción alérgica además de mastocitos, basófilos y eosinófilos, los linfocitos TCD4 tipo Th2 tenían un papel fundamental. Estos linfocitos producen las citoquinas mediadoras de la reacción alérgica como IL-4 que induce la producción de la inmunoglobulina $E$ (IgE) especifica frente a alérgenos, IL-5 que induce la proliferación, diferenciación y activación de eosinófilos y la IL-13 que tiene actividad inmunomodulatoria y se sobrepone con la IL-4 en la producción de $\operatorname{Ig} E^{2}$.

A pesar de la aparición de las guías clínicas para diagnostico manejo y tratamiento del asma bronquial y la introducción de anti-inflamatorios (corticoides inhalados) en el tratamiento del asma bronquial hace ya 30 años, aún hay pacientes que mueren a causa de esta enfermedad ${ }^{3}$, y los costos en tratamiento han experimentado un gran aumento sin observarse un significativo progreso en los resultados de los tratamientos. Un grupo de expertos investigadores y clínicos formó una comisión para identificar aquellas áreas que se han estancado en el manejo de esta patología ${ }^{4}$. Uno de los argumentos es que el diagnóstico basado en la función debería ser implentado con el uso de biomarcadores del tipo de inflamación que puedan ser medidos, y de esta manera categorizar los fenotipos y endotipos de los pacientes asmáticos y realizar tratamientos personalizados. Así, con características clínicas, de función y respuesta a tratamiento se ha evidenciado que el asma bronquial es una enfermedad compleja que tiene diferentes expresiones o fenotipos.

Ya en 1927 Francis Rackemann se refiere a la heterogeneidad del asma y la clasifica en asma extrínseca (alérgica) e intrínseca (no alérgica). Por su parte, el informe 2019 de GINA (Global Initiative for Asthma menciona 5 fenotipos de asma, además de los fenotipos de asma alérgica y no alérgica incluye los fenotipos de 'asma de inicio tardio', 'asma con persistente limitación del flujo aéreo' (de larga evolución con remodelación de la vía aérea) y 'asma con obesidad'.

La definición de fenotipo en biología es el conjunto de caracteres visibles que un individuo presenta como resultado de la interacción entre su genotipo y el medio. Fenotipos son las características

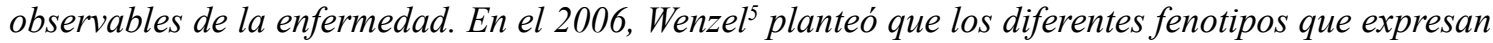
los pacientes adultos con asma son procesos diferentes en cada individuo, por lo tanto el diagnóstico de asma abarca muchas variantes de la enfermedad con diferentes etiologías y fisiopatologías. El endotipo ${ }^{6}$ es una vía biológica que define una etiología y que se define por un mecanismo funcional o fisiopatológico diferente. Son una forma diferente de clasificación que la de los fenotipos. Así, cada 
endotipo puede abarcar varios fenotipos, como también ciertos fenotipos están presentes en varios endotipos ${ }^{7}$. Con esta caracterización se plantea poder identificar nuevas vías biológicas y marcadores que permitan un mejor diagnóstico, pronóstico y tratamiento de los pacientes con asma bronquial ${ }^{8}$.

La clasificación en grupos de pacientes con características clínicas, funcionales, evolución en el tiempo y respuesta al tratamiento, ha sido de gran ayuda para conocer las diferencias de algunas asmas de evolución grave comparadas con aquellas de evolución leve o moderada que tienen buena respuesta al tratamiento convencional. Siendo la inflamación la característica inmunológica más importante en la patogenia del asma bronquial, la inflamación eosinofilica, ha sido la más estudiada y la que se asocia al asma extrínseco causada por respuesta a alérgenos comunes. Estos fenotipos de asma que presentan una respuesta inmune características de linfocitos CD4+ tipo Th2 es actualmente conocido como T2 alta que generalmente se presenta en pacientes pediátricos y en alrededor de un $60 \%$ en adultos ${ }^{9}$. Esta respuesta $T 2$ alta de inmunidad adaptativa presenta IgE especifica a alérgenos en suero, resultado de la presentación de alérgenos por células dendriticas presentadoras de alérgenos (antígenos) a las células Th2, las que al activarse producen citoquinas como las interleuquinas (IL): IL-4, IL5, IL-13. Estas citoquinas propias de la reacción alérgica inducen eosinofilia en sangre y vía aérea y biomarcadores dependientes de las células epiteliales de la vía aérea que expresan receptores IL-4 Ra para IL4y/o IL13 como periostin en suero y óxido nítrico en el aire exhalado ${ }^{10}$. Además de esta respuesta de inmunidad adaptativa, la inmunidad innata representada por los linfocitos innatos tipo 2 (ILC2), basófilos y mastocitos producen ILs tipo 2 como IL-4, IL5,IL-9 e IL-13. Esta respuesta que ha sido estudiada en pacientes ancianos que presentan eosinofilia en sangre y vía aérea y concentración de IgE policlonal sérica relativamente baja, se presenta como asma severa con mala respuesta a tratamiento esteroidal y mayor frecuencia de rinosinusitis crónica y pólipos nasales. El papel de los ILC2 se ha estudiado en pacientes con pólipos nasales y con reacción de sensibilidad a anti-inflamatorios no-esteroidales ${ }^{11}$. Los ILC2 pueden ser activados por ILs producidas por células epiteliales como IL-23, IL-25, IL-1 y TSLP (Thymic stromal Lymphopoietin) considerada una citoquina IL-7 símil. Estos ILC2 presentan receptores para prostaglandina $D_{2}\left(P G D_{2}\right)$ denominados CRTH2 y producen IL-9 que junto a IL-13 inducen metaplasia de células caliciformes.

En los últimos años se ha dado gran importancia al papel del epitelio no solo como centinela de la respuesta inmune innata, sino como en su rol frente a la respuesta a virus, contaminantes ambientales, humo de cigarrillo y alérgenos que pueden exacerbar y perpetuar el asma bronquial ${ }^{12}$. El epitelio bronquial expresa receptores de reconocimiento conocidos como Toll-like (TLR) como TLR4 y receptores activados frente a proteasas: PAR2 que responden a diferentes estímulos. Al activarse las células del epitelio producen IL-1, IL-33, TSLP, GM-CSF (factor estimulador de colonias de granulocitos y macrófagos) , M-CSF (factor estimulante de colonias de macrófagos), TGF- $\beta$ (factor de crecimiento transformador - beta). Estas citoquinas pueden activar células dendríticas promoviendo la respuesta inmune adaptativa y también las células de respuesta inmune innata como linfocitos ILC2, basófilos, mastocitos y eosinófilos que producen más citoquinas y mediadores inflamatorios contribuyendo al proceso inflamatorio y daño del asma bronquial. Cuando un paciente con asma alérgica es sometido a una provocación con alérgeno mediante broncoscopía, montan una respuesta aguda de inmunidad innata con producción de IL-33, IL-25, TSLP y GM-CSF ${ }^{13}$ esto implica que la provocación con antígeno inducen en las células epiteliales la producción de interleuquinas de respuesta innata en pacientes atópicos y que se correlacionan con el aumento de obstrucción bronquial en la fase tardía lo que indica que estas ILs son moléculas potenciales para inhibir la inflamación y obstrucción de la vía aérea por alérgenos.

Otro fenotipo de asma descrito es el asma neutrofilica caracterizada por aumento de neutrófilos en la vía aérea. La mayoría de los pacientes pueden presentar aumento de neutrófilos en la vía aérea durante una exacerbación, pero solo puede reflejar la respuesta a la infección viral causante del aumento de neutrófilos. Hay un grupo de pacientes con asma severa que presentan aumento de eosinófilos y neutrófilos en la vía aérea no estando con una exacerbación y generalmente son resistentes al tratamiento esteroidal ${ }^{14}$. El mecanismo por el cual se produce este aumento de neutrófilos en la vía aérea no se conoce bien, ya que hay múltiples mediadores incluyendo citoquinas y quemoquinas, humo de cigarrillo, comorbilidades como reflujo gastroesofágico y obesidad que se han relacionado con el aumento de neutrófilos en la vía aérea. 
Estudios de seguimiento en niños desde lactantes hasta la adolescencia han mostrado diferentes fenotipos de sibilancias que han sido homologadas al diagnóstico de asma bronquial ${ }^{9}$. En un trabajo reciente ${ }^{10}$ se enlazó varias cohortes con seguimientos desde lactante hasta la adolescencia y se concluye que habría 5 fenotipos de evolución de las sibilancias incluyendo un 50\% de niños que nunca sibila. Un 9\% presenta sibilancias tempranas hasta los 3-5 años y comienza a remitir en el periodo escolar, un 7\% es de presentación tardía comienza alrededor de los 3 años y persiste hasta la adolescencia. Un $24 \%$ presenta sibilancias desde lactante y remite en la edad pre-escolar o escolar y un $8 \%$ es de presentación temprana y persiste hasta la adolescencia siendo el grupo que se asocia con asma bronquial. Entender los mecanismo patogénicos del asma bronquial en los niños, es aún más complejo que en los adultos, ya que debe ser examinada en relación al desarrollo y maduración de su sistema respiratorio.

La expresión génica y regulación epigenética cambia con la edad y puede variar con diferentes exposiciones ambientales. Asi el lumen de la vía aérea y los volúmenes pulmonares aumentan hasta la adolescencia, mientras el desarrollo del sistema inmune se completa alrededor de los 5 años de edad. Esto puede determinar la sintomatología presente en diferentes edades.

Durante esta jornada de la SER pudimos constatar que en los últimos años se han producido grandes avances en el conocimiento de la fisiopatología del asma bronquial determinantes para tener un tratamiento mejor y más personalizado. Sin embargo, tenemos que recordar que en el $80 \%$ de los pacientes con asma, su enfermedad es leve a moderada por lo que pueden ser tratados con terapias convencionales.

Dra. Patricia Díaz Amor ${ }^{1}$

${ }^{1}$ Coordinadora, $6^{a s}$ Jornadas de Primavera Sociedad Chilena de Enfermedades Respiratorias Email: patriciadiazamor@gmail.com

\section{Bibliografía}

1.- GLOBAL INITIATIVE FOR ASTHMA (GINA) 2019 [GINA-2019-main-report-June-2019-wms- pdf] Disponible en: https://ginasthma.org/wp-content/ uploads/2019/06/GINA-2019-main-report-June2019-wms.pdf Consultado: 4.10.2019.

2.- DÍAZ P. Citoquinas: La internet biológica. ¿Futura terapia en el asma bronquial? Rev Chil Enf Respir 2001; 17: 32-42.

3.- EBMEIER S, THAYABARAN D, BRAITHWAITE I, BENAMARA C, WEATHERALL M, BEASLEY R. Trends in international asthma mortality: analysis of data from the WHO Mortality Database from 46 countries (1993-2012). Lancet 2017; published online Aug 7. doi:10.1016/S0140-6736(17)31448-4.

4.- PAVORD ID, BEASLEY R, AGUSTI A, ANDERSON GP, BEL E, BRUSSELLE G, et al After asthma: redefining airways diseases The Lancet Commissions www. thelancet.com September 2017.

5.- WENZEL SE. Asthma: defining of the persistent adult phenotype Lancet 2006; 368: 804-13.

6.- ANDERSON G. Endotyping asthma: new insights into key pathogenetic mechanisms in a complex heterogeneous disease. Lancet 2008; 372: 1107-19.

7.- LÖTVALL J, AKDIS CA, BACHARIER LB, BJERMER L, CASALE TB, CUSTOVIC A, et al. Asthma endotypes: A new approach to classification of disease entities within the asthma syndrome J Allergy Clin Immunol 2011;127:335-60.

8.- FAJT ML, WENZEL SA. Asthma phenotypes and the use of biologic medications in asthma and allergic disease: The next step to personalized care. J Allergy Clin Immunol 2015; 135: 299-310.

9.- FAHY JV. Type 2 inflammation in asthma present in most, absent in many Nat Rev Immunol 2015; 15: 57 65.

10.- JIA G, ERICKSON RW, CHOY DF, MOSESOVA S, WU LC, SOLBERG OD, et al. Bronchoscopy exploratory research study of biomarkers in corticosteroid refractory asthma (BOBCAT) Study group. Periostin is a systemic biomarker of eosinophilic airway inflammation in asthmatic patients J Allergy Clin Immunol 2012; 130: 647-54.

11.- SALIMI M, STÖGER L, LIU W GO S, PAVORD I, KLENERMAN P, et al. Cysteinyl leukotriene E4 activates human group 2innate lymphoid cells and enhances the effect prostaglandin D2 and epithelial cytokines J Allergy Clin Immunol 2017; 140: 1090-100.

12.- HOLGATE ST. The sentinel role of the airway epithelium in asthma pathogenesis Immunol Rev 2011; 242:205-19

13.- WANG W, LI Y, LV Z, CHEN Y, LI Y, HUANG K, CORRIGAN CJ, et al. Bronchial allergen challenge 
of patients with atopic asthma triggers an alarmin (IL33 ,TSLP, and IL-25) response in the airways epithelium and submucosa J Immunol 2018; 2221-31.

14.- MARTÍNEZ FD, WRIGHT AL, TAUSSIG LM, HOLBERG CJ, HALONEN M, MORGAN WJ. The group Health Medical Associates. Asthma and wheezing in the first year of life. M Engl J Med 1995; 332: 133-8.

15.- OKSEL C, GRANELL R, HAIDER S, FONTANELLA S, SIMPSON A, TURNER S, et al. Distinguishing wheezing phenotypes from infancy to adolescence. A pooled analysis of five birth cohorts Ann Am Thoracic Soc 2019; 16: 868-76. 